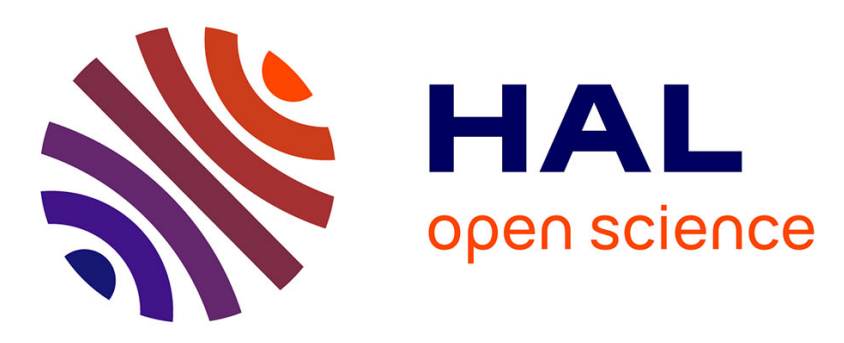

\title{
Driven-Voltage Permeability Variation Measurements of Bilayered Magnetostrictive/Piezoelectric Materials \&for Tunable Microwave Applications
}

Serge de Blasi, Patrick Queffelec, Sébastien Dubourg, Olivier Bodin, Marc Ledieu

\section{To cite this version:}

Serge de Blasi, Patrick Queffelec, Sébastien Dubourg, Olivier Bodin, Marc Ledieu. DrivenVoltage Permeability Variation Measurements of Bilayered Magnetostrictive/Piezoelectric Materials \&for Tunable Microwave Applications. IEEE Transactions on Magnetics, 2007, 43 (6), pp.2651. 10.1109/TMAG.2007.893789 . hal-00172013

\section{HAL Id: hal-00172013 https://hal.science/hal-00172013}

Submitted on 13 Sep 2007

HAL is a multi-disciplinary open access archive for the deposit and dissemination of scientific research documents, whether they are published or not. The documents may come from teaching and research institutions in France or abroad, or from public or private research centers.
L'archive ouverte pluridisciplinaire HAL, est destinée au dépôt et à la diffusion de documents scientifiques de niveau recherche, publiés ou non, émanant des établissements d'enseignement et de recherche français ou étrangers, des laboratoires publics ou privés. 


\title{
Driven-Voltage Permeability Variation Measurements of Bilayered Magnetostrictive/Piezoelectric Materials \&for Tunable Microwave Applications
}

\author{
S. De Blasi ${ }^{1}$, P. Quéffélec ${ }^{1}$, S. Dubourg ${ }^{2}$, O. Bodin ${ }^{2}$, and M. Ledieu ${ }^{2}$ \\ ${ }^{1}$ LEST-UMR CNRS 6165, Université de Bretagne Occidentale, Brest Cedex 393837-29238, France \\ ${ }^{2}$ CEA, Le Ripault, Monts 37260, France
}

\begin{abstract}
The complex permeability variation measurements at microwave frequencies of a magnetostrictive/piezoelectric bilayer under different values of a dc electric field are reported. A $25 \%$ variation of the initial permeability is achieved under a $1.5 \times 10^{6} \mathrm{~V} / \mathrm{m}$ static electric field.
\end{abstract}

Index Terms-Ferromagnetic materials, magneto-mechanical effects, microwave tunable devices, piezoelectric materials.

\section{INTRODUCTION}

$\mathbf{T}$ HERE is a great number of wireless communication applications working in the decimeter wave range, and the question a user may legitimately ask is whether or not his mobile phone will be able to operate with the different standards or if it will be necessary to get different systems each working at a specific frequency to cover the whole range. One solution is a single device incorporating various components, each working at a fixed frequency, but the best solution is a device incorporating microwave components able to work at various frequencies. Tunability which consists of modifying the transmission characteristics (center operating frequency and/or pass-bandwidth) thanks to an external command (static current or voltage) was previously achieved by inserting active components [1]. But, because of the well-known limitations of this approach [low signal-to-noise ratio (SNR)], substitute technologies like MEMS [2] and dielectric substrates exhibiting a variable permittivity such as ferroelectrics [3] have been studied. In the same way, a sensitive tunability was performed by inserting magnetic/dielectric composites into a transmission line under a weak static magnetic field [4], [5]. However, the integration of the magnetic control device (coils) is not compatible with the miniaturization of microwave circuits. Ferromagnetic materials would be good candidates for tunable applications at microwave frequencies, provided a new kind of static command can be developed. Fortunately, it is well known that the magnetic properties of a material under stress can also be varied (reverse magnetostrictive effect). The present paper aims at measuring how much the magnetic properties, such as the permeability spectra (the permeability magnitude and the gyromagnetic resonance frequency), of a ferromagnetic layer are modified at microwave frequencies, when the piezoelectric substrate on which it lies is strained under the action of a static electric field (reverse piezoelectric effect).

Digital Object Identifier 10.1109/TMAG.2007.893789

Color versions of one or more of the figures in this paper are available online at http://ieeexplore.ieee.org.

\section{Electric Field Sensitivity of A Magnetic Material}

\section{A. Inverse Magnetostrictive Effect}

A uniaxial stress $\sigma_{x}$ applied to a magnetic layer induces an equivalent stress-induced anisotropy field $H_{a \sigma}$ [6]. It can be defined as the value of the static magnetic field necessary to rotate in its direction the moments of a magnetic sample under stress

$$
H_{a_{\sigma}}=\frac{2 K_{1}-3 \lambda_{s} \sigma_{x}}{\mu_{0} M_{s}}
$$

where $M_{S}$ is the saturation magnetization, $\mu_{0}$ the permeability of vacuum, $\lambda_{S}$ the magnetostriction constant, and $K_{1}$ the magneto-crystalline anisotropy energy. Then, the initial magnetic susceptibility can be expressed as

$$
\chi_{0 \sigma}=\frac{\partial M}{\partial H}=\frac{\mu_{0} M_{S}^{2}}{2 K_{1}-3 \lambda_{s} \sigma_{x}} .
$$

The equation of motion without damping of a magnetic moment under the effective dc field $H_{a \sigma}$ [7] gives rise to an expression of the high frequency susceptibility

$$
\chi(f)=\frac{\chi_{0 \sigma}}{1-\left(f / f_{0}\right)^{2}}
$$

with $f_{0}=\gamma \sqrt{H_{a \sigma}\left(H_{a \sigma}+4 \pi M_{S}\right)}$, where $\gamma$ is the gyromagnetic ratio.

Even if (3) predicts a variation under stress of the rf spin permeability in the whole frequency range, we will only consider in the next section the static permeability, because its variations implies a shift of the real part of the permeability spectrum below the gyromagnetic absorption region, which is aimed for magnetic-based tunable applications.

\section{B. Inverse Piezoelectric Effect}

The inverse piezoelectric effect [8] corresponding to the strain $e_{h}$ induced by a piezoelectric material under a static applied electric field $E_{m}$ can be expressed along the $x$ axis as

$$
e_{x}=d_{11} E_{x}+d_{21} E_{y}+d_{31} E_{z}
$$

where the $d_{i j}$ are the piezoelectric coefficients, when no external stress is applied to the material. 
In the case of a static electric field applied in the $z$ direction

$$
e_{x}=d_{31} E_{z}
$$

and

$$
\sigma_{x}=Y d_{31} E_{z}
$$

where $Y$ is the Young's modulus in the $(x, y)$ plane and $d_{31}$ the deformation in the $x$ direction when an electric field is applied in the $z$ direction.

\section{Coupled Inverse Magnetostrictive/Piezoelectric Effects}

If a magnetic layer set onto a piezoelectric substrate is biased by a static electric field, its properties will vary because of the stresses induced by the substrate deformation due to the inverse piezoelectric effect. Indeed, if (5) is inserted into (1), it follows that an equivalent electrical anisotropy field $H_{a E}$ can be expressed as

$$
H_{a_{E}}=\frac{2 K_{1}-3 \lambda_{s} Y d_{31} E_{z}}{\mu_{0} M_{s}} .
$$

In the same way, if (5) is inserted into (2), the magnetic susceptibility becomes

$$
\chi_{0 E}=\frac{\partial M}{\partial H}=\frac{\mu_{0} M_{S}^{2}}{2 K_{1}-3 \lambda_{s} Y d_{31} E_{z}}
$$

and the electric field sensitivity of the magnetic layer is

$$
\frac{\partial \chi_{0 E}}{\partial E}=\frac{3 \lambda_{s} Y d_{31} \mu_{0} M_{s}^{2}}{\left(2 K_{1}-3 \lambda_{s} Y d_{31} E_{z}\right)^{2}} .
$$

With the equation above, if the magnetic material has the same Young's modulus and thickness as the piezoelectric substrate, the initial static susceptibility variation of a magnetic material, lying on a piezoelectric substrate under a static electric field, can be evaluated.

\section{EXPERIMENTAL SETUP}

\section{A. Piezoelectric Substrate}

Rectangular pieces $\left(20 \mathrm{~mm}^{2}\right)$ of soft tetragonal Lead-Zirconate-Titanate (PZT) were cut in a $z$-poled PPK-21 $100-\mu$ m-thick piezoelectric substrate (STELCO GmbH). Silver pasted, screen printed, and burnt electrodes had been previously deposited on both sides of the substrate. This material offers great longitudinal $d_{33}=500 \mathrm{pC} / \mathrm{N}$ and transverse $d_{31}=-220 \mathrm{pC} / \mathrm{N}$ piezoelectric constants, has a Curie temperature $\theta_{c}=230^{\circ} \mathrm{C}$, and has a Young's modulus $Y=65.10^{9} \mathrm{~N} / \mathrm{m}^{2}$.

\section{B. Ferromagnetic Layer}

A soft ferromagnetic material will be preferred for microwave tunable applications because of its low coercive field, its high saturation magnetization to work over a wide frequency range (the gyromagnetic frequency $f_{r} \propto 4 \pi M_{s}$ ), and its high static permeability allowing a significant shift under an external static

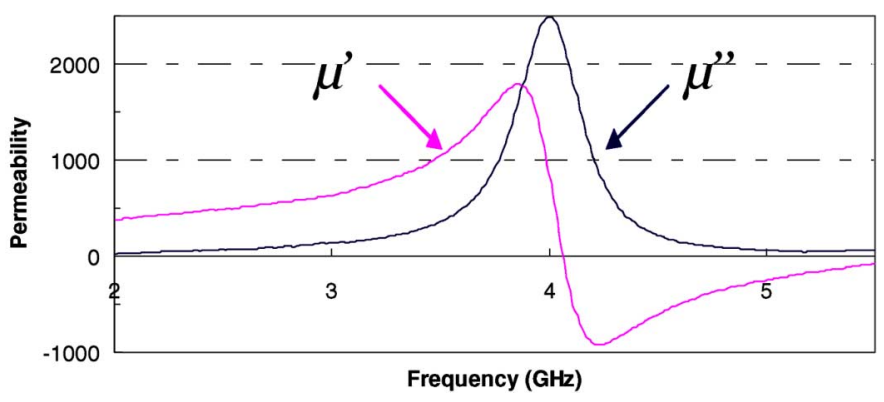

Fig. 1. Measured permeability spectra of 140-nm FeCoSiB deposition on thick smooth glass substrate.

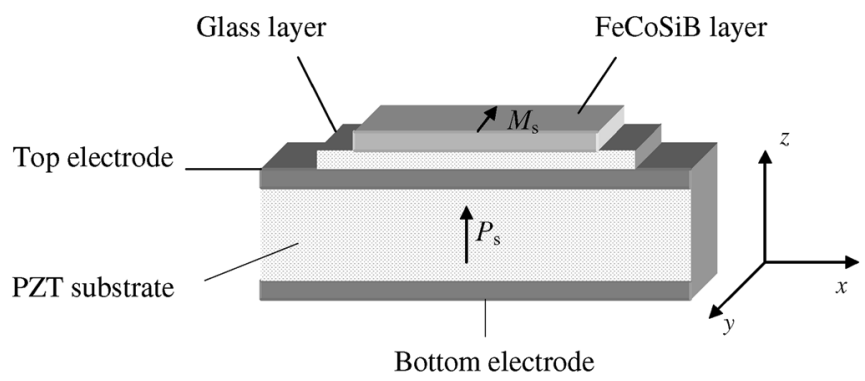

Fig. 2. Structure of magnetostrictive/piezoelectric composite.

command. Moreover, because the reverse magnetostrictive effect is to be used, the magnetic material must exhibit a high magnestostriction constant. Finally, because ferromagnetics are conductive materials, their thicknesses must also be thinner than the skin depth to avoid losses due to induced eddy currents. A 140-nm-thin $\mathrm{Fe}_{66} \mathrm{Co}_{18} \mathrm{Si}_{2} \mathrm{~B}_{14}$ ferromagnetic amorphous layer with $\lambda_{s}=3510^{-6}$ was first deposited by magnetron sputtering on a thick smooth glass substrate. We measured a $4 \pi M_{s}=$ $17800 \mathrm{G}$, an anisotropy field $H_{k}=120 \mathrm{Oe}$, and a resistivity $\rho=140 \mu \Omega \mathrm{cm}$.

The permeability spectra show a natural (without external bias) gyromagnetic resonance at $4 \mathrm{GHz}$. A damping parameter $\alpha \approx 0.005$ was estimated. The real part of the permeability is close to 500 , there are no contributions of domain wall movements (Fig. 1) and the imaginary part of the permeability (losses) is weak at $f=2 \mathrm{GHz}$. Thus, this magnetic material can be used in tunable telecommunication devices working near this frequency (wireless applications).

\section{Ferromagnetic/Piezoelectric Composite}

A 30- $\mu \mathrm{m}$ glass substrate was bonded as an under layer onto the top Ag electrode of the PZT substrate (Fig. 2) because the deposition of the ferromagnetic layer directly onto the rough piezoelectric substrate, using the conductive magnetic material as an electrode, did not give interesting results. Then, the 140-nm-thin $\mathrm{Fe}_{66} \mathrm{Co}_{18} \mathrm{Si}_{2} \mathrm{~B}_{14}$ ferromagnetic amorphous layer was deposited by magnetron sputtering onto the glass under layer.

During the deposition, the samples were oriented to obtain a transverse easy axis of magnetization, and special care was taken not to exceed the Curie temperature of the PZT substrate to preserve its remanent polarization. 
(a)

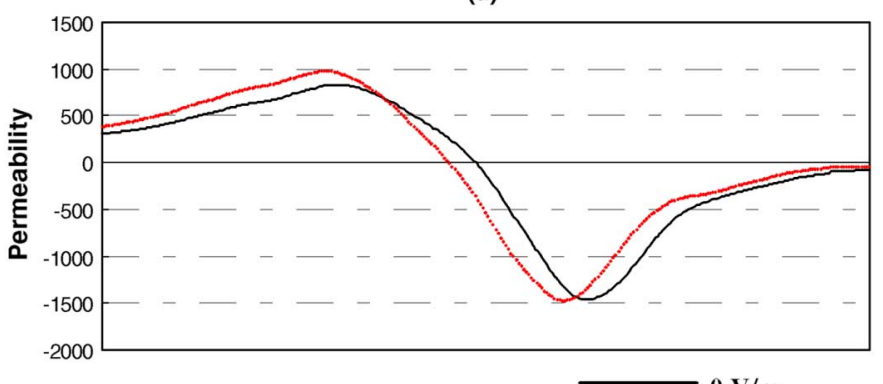

(b)

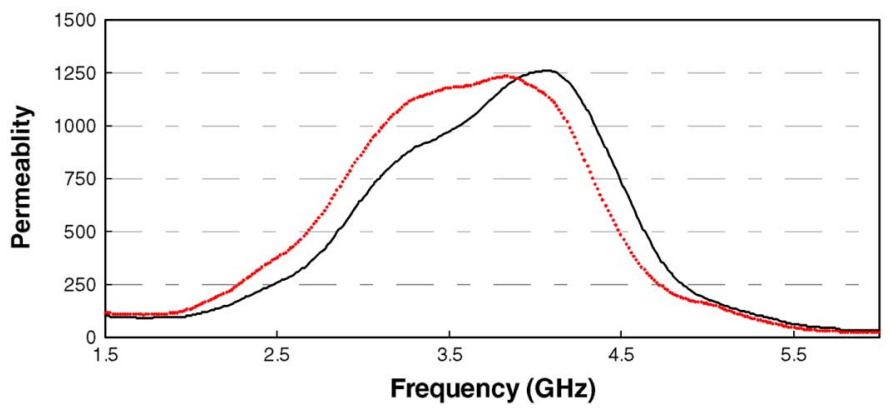

Fig. 3. Measured variations of (a) real part and (b) imaginary part of magnetostrictive/piezoelectric composite permeability under static electric field of $1.5 \times 10^{6} \mathrm{~V} / \mathrm{m}$.

\section{MEASUREMEnTS}

A permeameter based on the measurement of the impedance of an inductive loop [9] containing the polarized ferromagnetic/ piezoelectric composite to be characterized was used to demonstrate the variations of the real and imaginary parts of the permeability at microwave frequencies.

The frequency dependency of the losses plotted in Fig. 3(b), which corresponds to the magnetic material deposited as shown in Fig. 2, is less sharply defined than the one obtained with the glass substrate (Fig. 1). This difference can probably be explained by a nonuniformity of the local anisotropy field strength distribution, due to the higher roughness of the $30-\mu \mathrm{m}$-glass underlayer.

The real part of the permeability is increased of $25 \%$ at a frequency $f=2 \mathrm{GHz}$, and the resonance frequency is shifted from 4 down to $3.8 \mathrm{GHz}$ under the action of a $1.5 \times 10^{6} \mathrm{~V} / \mathrm{m}$ static electric field. These results are in good agreement with Snoek's law predicting an inverse relationship between the resonance frequency and the static permeability [10]. These variations are the consequence of a diminution of the net anisotropy in the transverse direction due to the strained piezoelectric substrate. According to (5), such a static electric field should correspond to an estimated net stress in the $x$ direction of $\sigma_{x}=50 \times 10^{6}$ $\mathrm{N} / \mathrm{m}^{2}\left(Y_{\text {ferro }} \approx 170 \times 10^{9} \mathrm{~N} / \mathrm{m}^{2}[11]\right)$. In fact, the deformation of the piezoelectric substrate yields a balanced biaxial stress in the $(x, y)$ plane inducing an anisotropy in the $(y, z)$ plane too, which decreases the stress induced anisotropy in the $(x, y)$ plane [12]. An electric field-induced uniaxial deformation of the PZT substrate would probably give greater permeability variations.

\section{CONCLUSION}

A 140-nm-thin ferromagnetic $\mathrm{Fe}_{66} \mathrm{Co}_{18} \mathrm{Si}_{2} \mathrm{~B}_{14}$ has been deposited on a soft $100-\mu \mathrm{m}$-thick PZT substrate, and a $25 \%$ variation of the magnetic layer permeability has been measured as a static electric field of $1.5 \times 10^{6} \mathrm{~V} / \mathrm{m}$ was polarizing the piezoelectric layer. By solving the problem of the insertion of a static magnetic command in electronic devices, this work restores ferromagnetic materials as very good candidates for the design of miniaturized microwave tunable devices for telecommunication applications. After a design optimization to get an uniaxial induced stress to perform the magnetic property variations, a future paper will show how optimized magnetostrictive/piezoelectric composites can be inserted in a transmission line to tune the characteristics of a microwave circuit.

\section{ACKNOWLEDGMENT}

This work was supported in part by the Brittany Region.

\section{REFERENCES}

[1] I. C. Hunder and J. D. Rhodes, "Electronically tunable microwave bandpass filter," IEEE Trans. Microw. Theory Tech., vol. 30, no. 9, pp. 1354-1360, Sep. 1982.

[2] E. Fourn, "MEMS switchable interdigital coplanar filter," IEEE Trans. Microw. Theory Tech., vol. 51, no. 1, pp. 320-324, Jan. 2003.

[3] V. Laur, A. Rousseau, G. Tanné, P. Laurent, S. Députier, M. Guilloux-Viry, and F. Huret, "KTa ${ }_{0.6} \mathrm{Nb}_{0.4} \mathrm{O}_{3}$ (KTN) ferroelectric thin films behavior at microwave frequencies for tunable applications," IEEE Trans. Ultrasonics, Ferroelectrics Frequency Control, vol. 53, no. 12, pp. 2280-2286, Dec. 2006.

[4] E. Salahun, P. Quéffélec, G. Tanné, A. L. Adenot, and O. Acher, "Correlation between magnetic properties of layered ferromagnetic/dielectric material and tunable microwave device applications," J. Appl. Phys., vol. 91, no. 8, pp. 5449-5455, Apr. 2002.

[5] E. Salahun, G. Tanné, P. Quéffélec, M. Le Floc'h, A. L. Adenot, and O. Acher, "Application of ferromagnetic composite in different planar tunable microwave devices," Microwave Opt. Technol. Lett., vol. 30, no. 4, pp. 272-276, Aug. 2001.

[6] J. D. Livingston, "Magnetomechanical properties of amorphous metals," Physica Status Solidi (a), no. 70, pp. 591-596, Dec. 1981.

[7] C. Kittel, "On the theory of ferromagnetic resonance absorption," Phys. Rev., vol. 73, no. 2, pp. 155-161, Jan. 1948.

[8] W. G. Cady, Piezoelectricity, revised ed. New York: Dover, 1964, vol. 2, pp. 182-190.

[9] O. Acher, J. C. Peuzin, J. L. Vezmeulen, A. Lucas, Ph. Baclet, and J. Kazandjoglou, "Direct measurements of permeability up to $3 \mathrm{GHz}$ of Co-based alloys," J. Appl. Phys., vol. 73, no. 10, pp. 6162-6164, May 1993.

[10] J. L. Snoek, "Dispersion and absorption in magnetic ferrites at frequencies above $1 \mathrm{Mc} / \mathrm{s}$," Physica XIV, no. 4, May 1948.

[11] P. T. Squire, D. Atkinson, and S. Atalay, "Magnetostrictive and magnetoelastic properties of rapidly quenched wire," IEEE Trans. Magn., vol. 31, no. 2, pp. 1239-1248, Mar. 1995.

[12] J. D. Livingston, "Stresses and magnetic domains in amorphous metal ribbons," Physica Status Solidi (a), no. 56, pp. 637-645, Jul. 1979.

Manuscript received October 31, 2006 (e-mail: serge.deblasi@univ-brest.fr). 